\title{
Ultrafast spectral-domain optical coherence tomography realized by parametric spectro-temporal analyzer
}

\author{
Chi Zhang ${ }^{1,2}$, Xiaoming Wei ${ }^{2}$, Yiqing $\mathrm{Xu}^{2}$, Jianbing $\mathrm{Xu}^{2}$, Luoqin $\mathrm{Yu}^{2}$, Bowen $\mathrm{Li}^{2}$, Sisi $\mathrm{Tan}^{2}$, Andy K. S. Lau ${ }^{2}$, \\ Xie Wang ${ }^{2}$, Xing $\mathrm{Xu}^{2}$, Kevin K. Tsia ${ }^{2}$, and Kenneth K. Y. Wong ${ }^{2 *}$ \\ ${ }^{l}$ Wuhan National Laboratory for Optoelectronics, Huazhong University of Science and Technology, 1037 Luoyu Road, Wuhan, China \\ ${ }^{2}$ Photonic Systems Research Laboratory, Department of Electrical and Electronic Engineering, The University of Hong Kong, Hong Kong. \\ Author email address: kywong@eee.hku.hk
}

\begin{abstract}
Performance of the spectral-domain optical coherence tomography is limited by its Ascan rate, namely the frame rate of spectrometer. In this paper, $60-\mathrm{MHz} \mathrm{A}$-scan rate is achieved by adopting a recently demonstrated parametric spectro-temporal analyzer.

OCIS codes: (110.4500) Optical coherence tomography; (190.4975) Parametric processes; (300.6530) Spectroscopy, ultrafast.
\end{abstract}

\section{Introduction}

Optical coherence tomography (OCT) has proven to be a powerful non-invasive cross-sectional imaging modality, and it is advantageous in terms of lateral resolution, owing to its short wavelength [1]. Recent breakthroughs in OCT have greatly improved its A-scan rate (or line rate) and stability, and even exceeding $\mathrm{MHz}$ is available, in the scheme of swept-source OCT [2]. However, this scheme requires complex swept-source for the interference to ensure the linear spectro-temporal sequence, and if it is not strictly linear, re-sampling and calibration of the time axis is also required before the Fourier transformation. Therefore, its stationary source requirement is more stringent than the earlier generation spectral-domain OCT (SD-OCT), though whose 100-kHz A-scan rate is limited by the speed of the CCD or CMOS cameras, as shown in Fig. 1(a) \& (b) [3]. Therefore, ultrafast spectrometer with high sensitivity is highly desired to improve the SD-OCT system.

Recently, another temporal method called, parametric spectro-temporal analyzer (PASTA), achieved 100-MHz frame rate with $0.03-\mathrm{nm}$ resolution and $-40-\mathrm{dBm}$ detection sensitivity [4,5]. It can be naturally applied in the SDOCT system, and introduces orders of magnitude improvement. In this paper, by introducing this ultrafast PASTA system, the SD-OCT system has achieved $60-\mathrm{MHz}$ frame rate, and 150- $\mu \mathrm{m}$ resolution through the 9-nm observation bandwidth. Moreover, higher detection sensitivity has been demonstrated by increasing the averaging times. This scheme provides a promising solution for the ultrafast tomography applications.

2. Principle and experimental setup

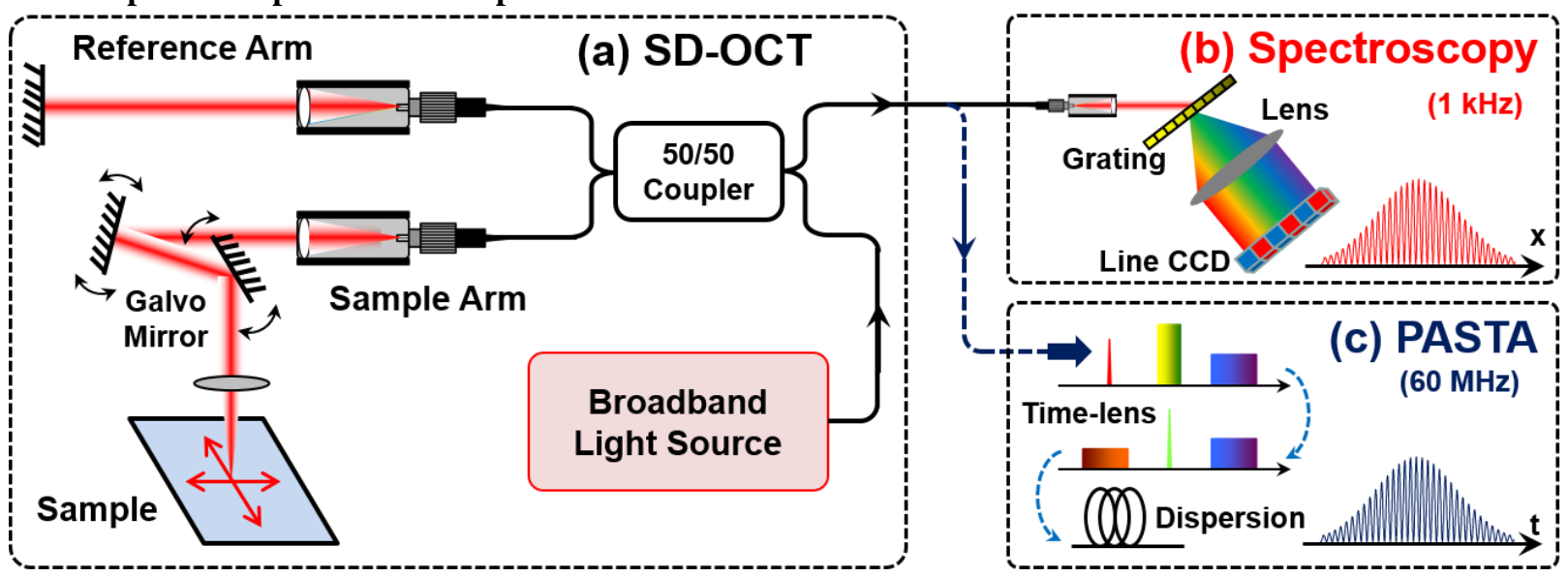

Fig. 1. Experimental setup of the SD-OCT system. (a) The interferometer part, the depth information of the sample is encoded as different fringes on the spectrum. (b) The spatial grating based spectrometer resolves these fringes through a spatial line CCD. (c) Temporal dispersion based PASTA system resolves these fringes through a time-lens focusing mechanism in the time domain.

Figure 1 shows the experimental setup of the SD-OCT. Here the broadband light source was provided with an incoherent amplified spontaneous emission (ASE) noise, from a pumped Erbium doped fiber (EDF). A 50/50 coupler splits the broadband source into the reference arm and sample arm separately; therefore, different sample depth will introduce spectral interference. There are two ways to capture these fringes: first, it is the most 
conventional spectrometer based on a spatial grating, while the CCD/CMOS sensor limits its frame rate to $1 \mathrm{kHz}$, as shown in Fig. 1(b); In the second scheme, the frame rate is greatly enhanced by the time-lens focusing mechanism, PASTA, and converted the wavelength information in the time axis, as shown in Fig. 1(c). The detailed configuration of the PASTA system is identical as that in the Ref. [4], except the repetition rate of the pump source is down-converted to $60 \mathrm{MHz}$ to enlarge its observation range (from $5 \mathrm{~nm}$ to $9 \mathrm{~nm}$ ).

\section{Results and discussions}

Due to the limited observation spectral bandwidth of PASTA $(9 \mathrm{~nm})$, this ultrafast SD-OCT system can only achieve $150-\mu \mathrm{m}$ resolution, which is a little bit degraded from the ideal $120 \mu \mathrm{m}$ [1]. Therefore, it is not sufficient to observe some bio-samples, and a reflective mirror was first employed to quantify its performance. To quantify its depth observation range, the mirror was moved across the depth (1-mm step across 12-mm range), to measure the roll-off curve as shown in Fig. 2(a). Owing to the incoherence of the broadband source, the signal-to-noise ratio (SNR) was only $20 \mathrm{~dB}$, which resulted in $\sim 60-\mathrm{dB}$ poor detection sensitivity. Therefore, longer accumulated time (or increased averaging times) was applied to improve the SNR, as shown in Fig. 2(b) \& (c). From these two figures, we can see that the SNR was improved to be $30 \mathrm{~dB}$ and $40 \mathrm{~dB}$, which corresponded to $80-\mathrm{dB}$ and $100-\mathrm{dB}$ detection sensitivity separately. Figure 2(d) - (o) illustrate the B-scan frames of the reflective mirror at different depths, to improve the imaging quality, each frame was averaged by 10 times, and still had a 6-MHz A-scan rate.
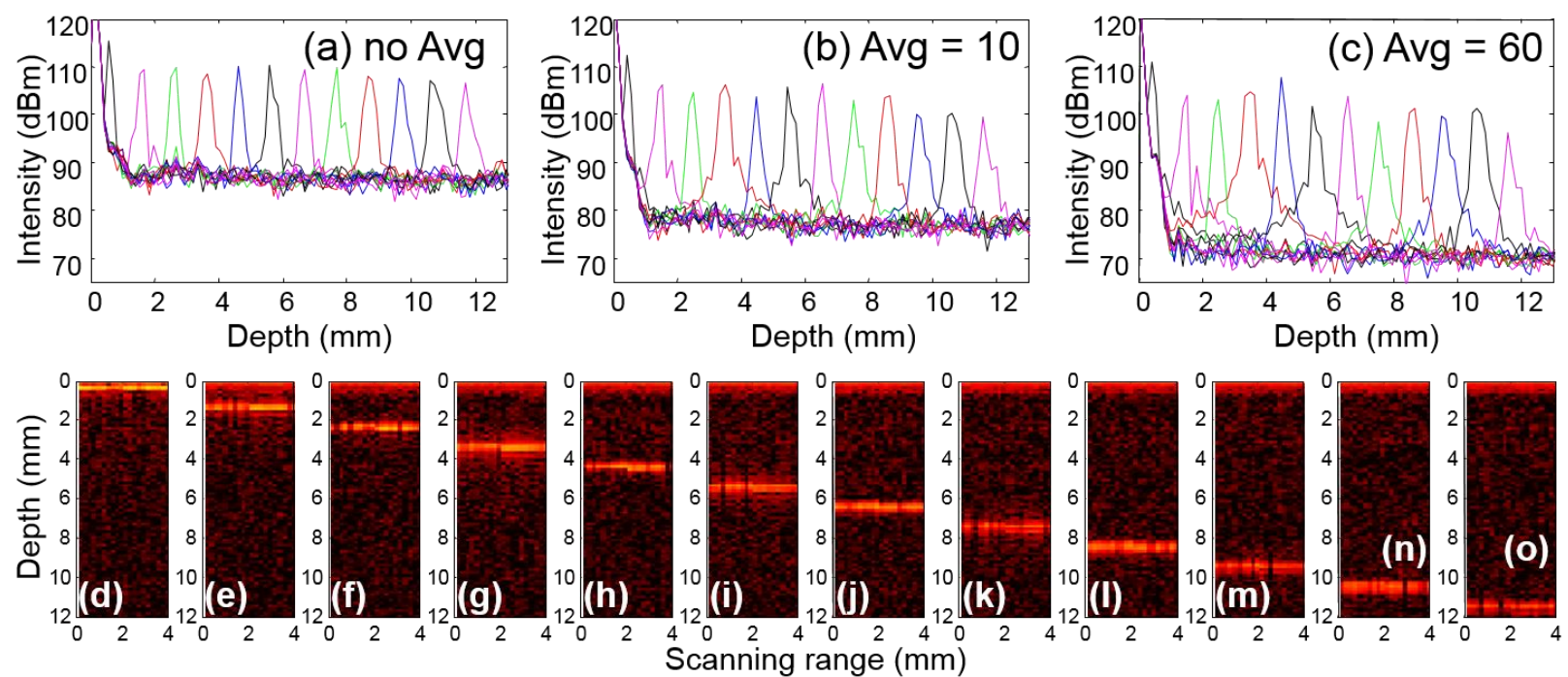

Fig. 2 Performance of the ultrafast SD-OCT image based on PASTA system. (a) - (c) The roll-off measurement of this ultrafast SD-OCT ( 0.5 $\mathrm{dB} / \mathrm{mm}$ ) with different averaging times. (d) - (o) The B-scan frames of the SD-OCT (averaged by 10 times) with the reflective mirror at different depths, which corresponds to positions of those roll-off curves in (a) - (c).

\section{Conclusion}

To sum up, we here employed a PASTA system to improve the frame rate of the conventional SD-OCT, and 60$\mathrm{MHz}$ A-scan rate was demonstrated, which was faster than the complex SS-OCT configuration [2]. However, owing to the limited observation spectral bandwidth of the PASTA, which is around 9-nm, the SD-OCT system can only achieve the resolution of $150 \mu \mathrm{m}$. Therefore, enlarging the PASTA bandwidth is the most critical part for this ultrafast SD-OCT scheme in practical use. Moreover, the detection sensitivity of the SD-OCT can be improved by averaging the PASTA frames, namely increasing the averaging times. Although an assorted of technological advancements have been demonstrated for scaling the A-scan rate of OCT, SD-OCT based on PASTA system provides a promising alternative solution for the tomography community.

Acknowledgment: The work was partially supported by grants from the Research Grants Council of the HKSAR, China (projects HKU 17208414 and HKU 717212E) and University Development Fund of HKU.

[1] D. Huang, et. al, “Optical Coherence Tomography," Science 254, 1178-1181 (1991).

[2] R.Huber, M. Wojtkowski, and J. G. Fujimoto, "Fourier Domain Mode Locking (FDML): A new laser operating regime and applications for optical coherence tomography," Opt. Express 14, 3225-3237 (2006).

[3] A. F. Fercher, et. al, "Measurement of intraocular distances by backscattering spectral interferometry," Opt. Commun. 117, 43-48 (1995).

[4] C. Zhang, et. al, "Parametric spectro-temporal analyzer (PASTA) for real-time optical spectrum observation," Sci. Rep. 3, 2064 (2013).

[5] C. Zhang, et. al, "Ultrafast optical spectrum observation based on telescope and wide-angle parametric spectro-temporal analyzer," Sci. Rep. 4,

5351 (2014). 